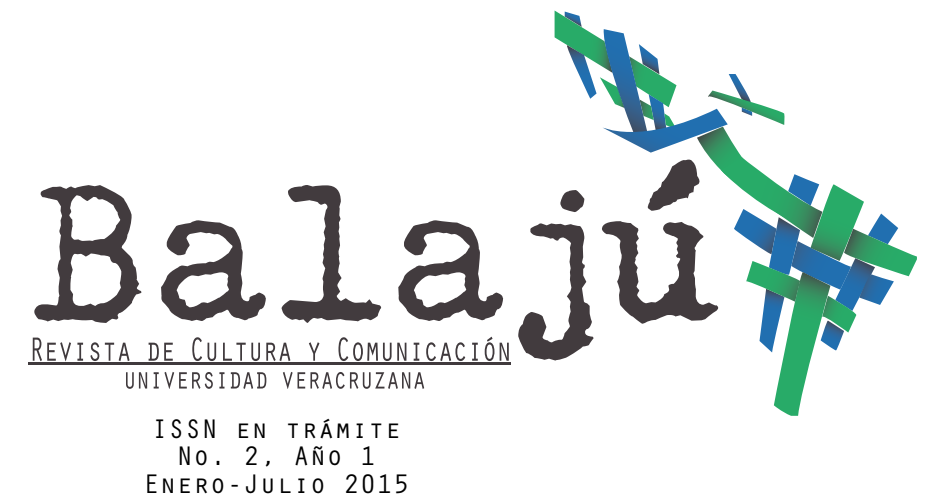

\title{
Serenata transnacional por la paz: Juliet Barrett Rublee y Flame of Mexico
}

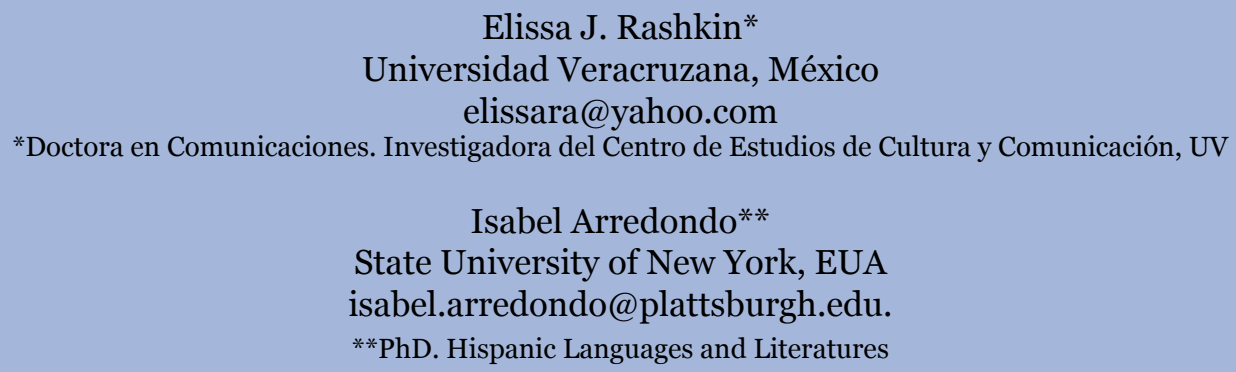

\section{RESUMEN}

Este artículo explora el mensaje de paz articulado por la activista feminista Juliet Barrett Rublee en Flame of Mexico( 1932), probablemente el primer largometraje de ficción estadounidense filmado en México y un texto híbrido en muchos sentidos. En particular se analiza la función de la música como discurso comunicacional multivalente.

\begin{abstract}
This article explores the pacifist message in feminist activist Juliet Barrett Rublee's Flame of Mexico (1932), probably the first full-length fiction film filmed by a United States crew in Mexico, and a hybrid text on many levels. We particularly focus on the multiple meanings conveyed by the film's musical accompaniment.
\end{abstract}

\section{PALABRAS CLAVES}

Juliet Barrett Rublee, Flame of Mexico, panamericanismo, historia del cine, feminismo transnacional.

\section{KEYWORDS}

Juliet Barrett Rublee, Flame of Mexico, pan-Americanism, film history, transnational feminism. 


\section{Serenata transnacional por la paz: Juliet Barrett Rublee y Flame of Mexico}

En abril de 1928, la feminista estadounidense Juliet Barrett Rublee llegó a México en compañía de su esposo George Rublee, abogado y asesor del embajador Dwight Morrow. Con una larga trayectoria en el feminismo de su país, la señora Rublee -activista, artista y entusiasta viajera- no tardó en encontrar inspiración, escribiendo el guion de lo que sería la película Flame of Mexico, considerada como el primer largometraje de ficción estadounidense rodado enteramente en México. Filmada en 1929, la película se desarrolla en vísperas de la Revolución y muestra la lucha por la tierra, destacando las injusticias sufridas por los campesinos a manos de capitalistas extranjeros, sobre todo de las compañías petroleras. Hecha en los albores del cine sonoro, la película originalmente fue muda; no obstante, en 1930-1932 Rublee agregó una banda sonora, creando así una cinta híbrida que narra su historia a través de títulos escritos, pero a la vez incluye música y sonido sincronizado².

La banda sonora de Flame of Mexico nos interesa por varias razones. Primero, porque muestra una familiaridad con la música popular mexicana por parte de Rublee, poco común entre intelectuales estadunidenses; de hecho, con la colaboración de Guty Cárdenas y la inclusión de piezas como la Canción mixteca en voz de Tito Guízar, entre otras, la película se asemeja más a los productos del emergente cine sonoro mexicano que a las cintas estadunidenses de la época. Esto se puede atribuir a la trayectoria de Rublee en la danza moderna, en el teatro neoyorquino y en el cosmopolita ambiente cultural de su época; sin embargo, aún más interesante es el uso que hace Rublee de estas canciones, ya que funcionan no sólo para representar y destacar las relaciones amorosas entre personajes de la película, sino como propuestas internacionalistas de paz entre las dos naciones.

Siendo Rublee una conocida pacifista y abogada por la justicia social en términos feministas e internacionalistas, consideramos que ella

1 Una versión de este texto fue presentada en el Congreso Internacional de Historia, Cuarto Encuentro del Grupo de Trabajo de la Asociación Europea de Historiadores Latinoamericanistas (AHILA), Veracruz, 15-17 de abril de 2015.

2 Flame of México: Productora y "supervisora" (de acuerdo con los créditos), Juliet Barrett Rublee; director, David Kirkland; fotografía, Jules Cronager; director técnico, Jorge Ahumada; intérpretes, Donald Reed (Rafael), Alicia Ortiz (Rosita), Francisco Martínez (Don Gonzalo de Aragón), Frank Hagney (Thornton), Jimmy Hodges (Fry). Los créditos musicales mencionamos más adelante y a lo largo del texto. 
utiliza la música como un elemento clave en la construcción del mensaje pro mexicanista y pro revolucionario que Flame of Mexico transmite de manera explícita. Este mensaje critica duramente la actuación de los empresarios estadunidenses en México y, por lo tanto, se aleja de la línea oficial de la embajada, aproximándose más a algunas posturas de la izquierda mexicana de la época. Nuestro artículo explora el innovador elemento musical de la comunicación fílmica plasmada en Flame of Mexico, considerando también la película como texto (trama, personajes, aspectos narrativos y discursivos) y su contexto histórico de producción, distribución y recepción. Argumentamos que la actuación de Rublee en México y la película que resultó de ella son hechos históricos poco conocidos pero sumamente interesantes e importantes en la historia de las redes transnacionales tejidas por activistas e intelectuales en las décadas de 1920 y 1930.

\section{Juliet Barrett Rublee, activista e internacionalista}

Juliet Barrett nació en 1875, en Chicago, Illinois's. Hija de familia acomodada, asistió a un colegio prestigioso para mujeres en Farmington, Connecticut. A los 24 años se casó con George Rublee, egresado de la Universidad de Harvard, quien se dedicó después a la carrera de relaciones internacionales. Durante la primera década de 1900, la pareja formó parte de la Cornish Art Colony en New Hampshire, una de las primeras colonias artísticas en Estados Unidos. Juliet tenía un gusto especial por el teatro y por la danza, y apareció en la obra ecologista Sanctuary: A Bird Masque de Percy Mac Kaye en 1913. Cabe notar que entre el elenco de dicha obra también estaban dos hijas del presidente Woodrow Wilson, quien asistió al estreno de la obra el 12 de septiembre, junto con su esposa $^{4}$. No obstante su interés y aparente éxito en este medio ${ }^{5}$, a partir de este momento las preocupaciones políticas de Rublee parecen haberla alejado del mundo de la danza, mientras su oposición a la participación estadounidense en la Primera Guerra Mundial y su creciente involucramiento en asuntos feministas la llevaron rápidamente a la esfera pública

3 Este apartado biográfico retoma la narrativa que elaboramos en Elissa Rashkin e Isabel Arredondo, "Juliet Barrett Rublee y la Revolución mexicana”, Susie Porter (coord.), Género en la encrucijada de la historia social e cultural.

4 La pareja presidencial también formó parte de la Cornish Colony, pasando ahí los veranos durante 1913-1915. 5 Una foto de Rublee aparece como frontispicio del libro The Book of the Dance de Arnold Genthe (Nueva York, Mitchell Kennerley, 1916), indicando su visibilidad en este campo de actividad artística. En marzo de 1926, Rublee volvió al ambiente escénico como productora de The Moon Is a Gong, obra teatral del novelista John Dos Passos, en la ciudad de Nueva York. 
como activista política.

El 9 de mayo de 1914, Rublee encabezó una marcha organizada por la Unión Legislativa por el Sufragio Femenino [Congressional Union for Woman Suffrage], en pro del voto femenino ${ }^{6}$. El siguiente año, participó en una delegación al Congreso Internacional de Mujeres a La Haya, representando la sección Washington D. C. del Partido de la Mujer por la Paz [Woman's Peace Party]. Fue una fuerte proponente de la no intervención estadounidense en la guerra europea; en 1917, desafió la desaprobación de su esposo George y protestó frente a la Casa Blanca a favor de la neutralidad 7 .

Un momento decisivo en la vida de Rublee sucedió en 1916 cuando conoció a Margaret Sanger, quien acababa de ser arrestada al abrir la primera clínica en Estados Unidos dedicada a la salud reproductiva de la mujer y al control de la natalidad, en Brooklyn, Nueva York ${ }^{8}$. A la detención de Sanger, Rublee fue en su defensa. Junto con otras mujeres ricas, estableció el Comité de 100 para recaudar fondos y atraer apoyo a la causa. Así Rublee y Sanger entablaron una estrecha amistad y Rublee empezó a jugar un papel prominente en el movimiento en pro de los derechos reproductivos, utilizando hábilmente sus conexiones con la alta sociedad para procurar recursos para la organización. En su autobiografía, Sanger destaca la importancia de Rublee para el movimiento, haciendo hincapié en su dedicación: "Nunca antes en un movimiento había una idealista más inspirada”, escribe. "La imaginación de esta pintoresca y romántica esposa de un abogado conservador había sido tan inflamada que dedicó a ella toda su devoción, lealtad y partidismo". Por otra parte, Ellen Chesler deja claro que Rublee no fue solamente "pintoresca y romántica", sino un actor clave cuyas redes sociales, poder económico y habilidades organizativas fortalecieron y hasta cambiaron la dirección del movimiento ${ }^{10}$.

En noviembre de 1921, Rublee fue una de las organizadoras del Pri-

\footnotetext{
6 "Suffragists Ask Congressfor Vote", New York Times, 10 de mayo de 1914: http://query.nytimes.com/mem/ archive-free/pdf?res=F70817F7355D13738DDDA90994DD405B848DF1D3 (consultado el 17 de febrero de 2015).

7 Según una anécdota contada a Paul Marashio por un sobrino de Rublee, George no quería que Juliet se manifestase en pro de la neutralidad de Estados Unidos en la Primera Guerra Mundial. El marido temía que el pacifismo de Juliet afectase su reputación como abogado. Por eso le dijo a Rublee: “¿Qué dirían si te arrestasen y dijesen 'La señora Rublee fue arrestada por manifestarse enfrente de la Casa Blanca?"” A lo que Juliet contestó: "George, no dirían eso. Dirían: 'Juliet Barret Rublee hizo esto' y eso no tiene nada que ver contigo". Al final, Rublee se manifestó en pro de la paz en 1917. Paul Marashio, "A Feminist Voice in New Hampshire”, p. 4. 8 Sobre Sanger y el movimiento en pro de los derechos reproductivos de la mujer, véanse Margaret Sanger, The Autobiography of Margaret Sanger; y Ellen Chesler, Woman of Valor: Margaret Sanger and the Birth Control Movement in America.
} 9 Margaret Sanger, Autobiography, p. 300.

10 Ellen Chesler, Woman of Valor, pp. 154, 167-168. 
mer Congreso sobre el Control de la Natalidad, un evento de tres días cuya reunión final fue interrumpida por la policía, resultando en numerosas detenciones. El 21 de noviembre, el New York Times publicó un largo pronunciamiento del arzobispo Patrick J. Hayes en contra del congreso, pero después, en el mismo artículo, otorgó a Rublee el derecho de réplica. Criticando enfáticamente las opiniones del arzobispo, Rublee declaró que "las leyes actuales no reflejan la voluntad del pueblo... Coincido con el arzobispo en que es correcto prevenir el envío por correo de literatura obscena e indecente, pero sostengo que la información científica que salvaguardará la salud de mujeres y niños no es ni obscena ni indecente" ${ }^{1}$. Sus comentarios en defensa del congreso y de la planificación familiar provocaron la detención de Rublee durante la audiencia judicial del 3 de diciembre $^{12}$. A pesar de su represión por las autoridades locales, el congreso marcó el inicio de la organización formal a favor del control de la natalidad, ratificado formalmente por el Tribunal Supremo de la Nación en abril de $1922^{13}$.

Al fundarse la Liga Estadounidense para el Control de la Natalidad [American Birth Control League, en adelante ABCL] en 1922, Rublee se convirtió en su vice-presidenta. Su papel protagónico en el movimiento puso a Rublee en contacto con mujeres y hombres de muchas partes del mundo -muy particularmente del estado de Yucatán-, donde el movimiento feminista florecía bajo el liderazgo de Elvia Carrillo Puerto, hermana del gobernador revolucionario Felipe Carillo Puerto y presidenta de la Liga Feminista Rita Cetina Gutiérrez. Fue por medio de estos lazos con las feministas yucatecas, en torno a la cuestión de la planificación familiar, que Rublee tuvo su primer contacto con la Revolución mexicana.

En 1923, Felipe Carrillo Puerto, a través de Ernest Gruening ${ }^{14}$, invitó a Sanger a Yucatán para desarrollar un proyecto de clínicas de control de la natalidad en Mérida. En una carta fechada el 3 de julio, Anne Kennedy,

11 “Hayes Denounces Birth Control Aim", New York Times, 21 de noviembre de 1921: http://query.nytimes. com/mem/archive-free/pdf?res=F20613FF3A5810738DDDA80A94D9415B818EF1D3 (consultado el 17 de febrero de 2015).

12 Rublee protestó que su detención fue ilegal ya que su único "delito" fue manifestar públicamente su desacuerdo con la sección del código civil prohibiendo la diseminación de información sobre el uso de anticonceptivos. "Mrs. Rublee Plans False Arrest Suit", New York Times, 6 de diciembre de 1921: http:/query.nytimes.com/ mem/archive-free/pdf?res=F6081EF63B5A1B7A93C4A91789D95F458285F9 (consultado el 17 de febrero de 2015).

13 “Birth Control Body Wins Charter Fight", New York Times, 14 abril 1922: http:/query.nytimes.com/mem/ archive-free/pdf?res=F40712FF3C5D1A7A93C6A8178FD85F468285F9 (consultado el 17 de febrero de 2015). 14 Gruening, editor de The Nation entre 1920 y 1923, fue proponente clave del control de la natalidad, y al mismo tiempo un observador agudo de los cambios sociopolíticos del México posrevolucionario, analizados en su clásico libro Mexico and Its Heritage. Entabló amistad con Felipe Carrillo Puerto y publicó varios libros y artículos sobre la vida, obra y muerte del gobernador yucateco. 
secretaria ejecutiva de la ABCL, contestó explicando que aunque Sanger no pudo aceptar la invitación, ella misma iría en agosto para explicar el trabajo de la League, demostrar los métodos y ayudar a formular el plan para el desarrollo de las clínicas locales ${ }^{15}$. Sin embargo, aunque el dato ha pasado algo desapercibido en las fuentes historiográficas, Kennedy no viajó sola sino en compañía de la vicepresidenta de la ABCL, Juliet Barrett Rublee.

La experiencia de Kennedy y Rublee en México fue documentada detalladamente en el artículo que Kennedy escribió después para la revista de la ABCL, Birth Control Review ${ }^{16}$. El artículo muestra que las dos activistas llegaron a Mérida el 15 de agosto, y pasaron 10 días en la región antes de partir a la Ciudad de México, donde pasaron otros 10 días. Aun antes de llegar, conocieron por azar en el barco a la esposa del gobernador Carillo Puerto, Isabel Palma, junto con un sobrino de aquél que hablaba inglés, quien les ayudó a conseguir entrevistas con "personas importantes" en Yucatán. Kennedy informa sobre su entrevista con el gobernador y elogia la política anticlerical de su administración, notando que "la única cuestión que ha estado en discusión es cómo llevar el Control de la Natalidad al pueblo, ya que extrañamente éste no está instruido en estos asuntos" ${ }^{11}$. En Mérida, ellas también se entrevistaron con un grupo de médicos asociados con el gobierno; de esta reunión surgió el plan de abrir dos clínicas de planificación familiar: una en el Hospital de Niños y Mujeres y la otra en la zona roja para atender a las trabajadoras sexuales ${ }^{18}$. Luego la secretaria del ABCL narra su encuentro con Elvia Carrillo Puerto, quien llevó con ella a la directora de parteras del hospital para recibir instrucción práctica sobre los métodos anticonceptivos.

En la Ciudad de México, Kennedy y Rublee se reunieron con diversos líderes sociales, entre ellos José Vasconcelos y Adolfo de la Huerta, en relación con el tema del control de la natalidad. Kennedy opinó que ahí, a diferencia de Yucatán, "las mujeres siguen bajo la dominación de los curas", lo cual explicaba la decisión del Congreso Panamericano de Mujeres, realizado unos meses antes en el mismo año de 1923, de aprobar clínicas de atención pre y posnatal, en lugar de promover abierta-

15 Monique J. Lemaître reproduce la carta de Kennedy en Elvia Carrillo Puerto. La Monja Roja del Mayab, p. 98

16 Anne Kennedy, "Birth Control in Mexico", en la sección "News Notes”, Birth Control Review, octubre de 1923, pp. 254-256. Agradecemos profundamente a Esther Katz y Cathy Moran Hajo del Margaret Sanger Papers Project (http://www.nyu.edu/projects/sanger/) por habernos proporcionado una copia de este artículo.

17 Anne Kennedy, op. cit., p. 255.

18 Estas clínicas, al parecer, nunca llegaron a ser realidad (Sarah A. Buck, "El control de la natalidad y el día de la madre: política feminista y reaccionaria en México, 1922-1923”, Signos históricos, p. 52). 
mente el uso de anticonceptivo ${ }^{19}$. Sin embargo, las dos representantes de la ABCL, optimistas, dejaron información para que fuese distribuida tanto en dichas clínicas como en consultorios particulares. A raíz de estas entrevistas, Kennedy concluyó que los dirigentes del país ("the few executive people of Mexico today") apoyaban firmemente el control de la natalidad como parte esencial del programa de reconstrucción nacional ${ }^{20}$.

Aunque aún no hemos encontrado ningún testimonio personal de Juliet Barrett Rublee sobre este viaje de 1923, no dudamos de que fuera un evento significativo en la formación de su pensamiento sobre la Revolución mexicana. De hecho, al regresar a México en 1928, Rublee reanudó su amistad con Elvia Carrillo Puerto, quien, a pesar del asesinato de su hermano y la consecuente represión de los grupos socialistas-feministas en Yucatán, seguía en el liderazgo del movimiento feminista a nivel nacional. Sus comentarios a Sanger constatan que la carismática dirigente feminista le impresionó sobremanera, y podemos especular que sus conversaciones le proporcionaron información e ideas sobre el feminismo, la revolución y las condiciones de las mujeres en el México posrevolucionario $^{21}$. En general, las actividades de Rublee en relación con el control de la natalidad, que desde 1923 le habían puesto en contacto con Carrillo Puerto y otros personajes importantes de la época, nos indican que la perspectiva que Rublee tenía sobre México no era precisamente el estereotipo citado por Emilio García Riera en su Historia documental del cine mexicano ${ }^{22}$, sino una extensión del compromiso personal y político de esta mujer culta e inteligente, quien desde años atrás había sido sumamente internacionalista en su pensamiento.

\section{Panamericanismo, feminismo y la campaña por la paz entre las naciones}

Las actividades internacionalistas de Juliet Rublee, culminando con la 19 Anne Kennedy, op. cit., p. 255.

$20 \mathrm{Ibid}$., p. 256. Por supuesto, las dos visitantes no podían imaginar que sólo cuatro meses después, los partidarios regionales de Adolfo de la Huerta se aprovecharían de la rebelión encabezada por éste para asesinar al gobernador Carillo Puerto y así acabar con los proyectos radicales y feministas en Yucatán, entre ellos, la promoción de los derechos reproductivos y el control de la natalidad.

21 Juliet Barrett Rublee, carta a Margaret Sanger, 22 de septiembre de 1928, Juliet Barrett Rublee Papers, Sophia Smith Collection, Smith College, Northampton, MA, 04: 622.

22 García Riera cita al periodista Campos Ponce quien, en el Diario del Sureste del 23 de febrero de 1937, caracterizaba a Rublee como "una millonaria yanqui" quien "ya anciana y enamorada de México, quiso propagar [en su película] lo bello de sus mujeres, de sus trajes y de sus bailes" (Historia documental del cine mexicano, tomo 1, Guadalajara, Universidad de Guadalajara/Gobierno de Jalisco/Conaculta/Imcine, 1992, p. 27). La designación de Rublee como "anciana" a sus 54 años de edad es sintomática del sexismo xenofóbico del texto original, repetido sin comentarios por el historiador. 
producción de su película Flame of Mexico a finales de la década de 1920, coincidieron con el movimiento feminista panamericanista explorado por la historiadora Megan Threlkeld en Pan American Women: U. S Internationalists and Revolutionary Mexico (2014) y otros textos. Según Threlkeld, la participación de mujeres estadounidenses -quienes, cabe recordar, aún no gozaban de los derechos plenos de la ciudadanía, siendo excluidas hasta 1922 del sufragio y por lo tanto de la representación política- en los debates sobre relaciones exteriores empezó a finales del siglo XIX; su enfoque inicial transatlántico dio paso, con la conclusión de la Primera Guerra Mundial, a un interés en las relaciones hemisféricas. Al ganar el derecho al voto, muchas veteranas de esa lucha dirigieron sus energías activistas hacia la política exterior ${ }^{23}$.

El trabajo de Threlkeld revela la estrecha conexión entre el feminismo -movimiento social amplio y diverso, enfocado sobre cuestiones de género y poder- y la política internacional; en primer lugar, las ahora ciudadanas procuraban apoyar a sus hermanas de otros países americanos, que también luchaban por el voto y otros derechos, lo cual exigía que las estadounidenses se educaran sobre las políticas públicas que mantenían otras naciones con respecto al sufragio, los derechos reproductivos y la regulación de la vida familiar y laboral de las mujeres. De manera que las panamericanistas, con el apoyo del gobierno estadunidense, organizaron congresos a los cuales invitaron a mujeres representantes de otros países.

El primer Congreso Panamericano de Mujeres [Pan American Conference of Women] se realizó en Baltimore, en 1922; asistieron más de 2 ooo delegadas representando a 23 naciones ${ }^{24}$. El segundo congreso se llevó a cabo en la Ciudad de México, en 1923, y en éste destacó la presencia de las feministas yucatecas agrupadas en la Liga Feminista Rita Cetina Gutiérrez; esta organización, promotora de los derechos reproductivos como hemos mencionado, editó en forma de panfleto un texto de Margaret Sanger titulado La regulación de la natalidad o la brújula del hogar (medios seguros y científicos para evitar la concepción), el cual se repartió tanto en Yucatán como en el Congreso Panamericano ${ }^{25}$. Las yucatecas también disintieron de las propuestas generalmente conservadoras pro23 Megan Threlkeld, Pan American Women: U. S. Internationalists and Revolutionary Mexico, p. 118. 24 Megan Threlkeld, “The Pan American Conference of Women, 1922: Successful Suffragists Turn to International Relations”, Diplomatic History, pp. 801-828; Julia Tuñón (comp.), Voces a lasmujeres. Antología del pensamiento feminista mexicano, 1873-1953, p. 246.

25 Monique J. Lemaître, op. cit., p. 89. Agradecemos a Stephanie J. Smith el haber compartido con nosotras su transcripción del panfleto de Sanger y otros materiales. Parte del folleto está reproducida en Tuñón (comp.), Voces a las mujeres, pp. 243-245. 
movidas por las feministas capitalinas en su crítica del matrimonio y el apoyo al llamado amor libre ${ }^{26}$. Cabe subrayar que esta postura más radical tenía mucho en común con el pensamiento personal y público de Rublee y de Sanger, defensoras de los derechos reproductivos de las mujeres y además, como su correspondencia personal indica, librepensadoras con respecto a una variedad de temas, desde la sexualidad hasta la telepatía, la salud y otros asuntos novedosos de su época ${ }^{27}$.

Cabe mencionar que, de acuerdo con Threlkeld y Ana LauJaiven, la delegación mexicana al congreso de 1922 en Baltimore, que incluía a Julia Nava de Ruisánchez y a Elena Torre, no limitó sus propuestas a asuntos convencionalmente femeninos o feministas, sino que también quería hablar sobre cuestiones de petróleo, tierras, asuntos obreros, migración y de la frontera ${ }^{28}$. No obstante, no lograron meter estos asuntos en la agenda general del congreso, y sólo los pudieron discutir en una reunión seccional. Las prioridades de las representantes mexicanas, al poner sobre la mesa la inversión económica e intervención política en México de parte de Estados Unidos, y las evidentes tensiones que provocaron debido a las diversas tendencias que constituían el movimiento de mujeres panamericanistas, reflejan las evidentes contradicciones en la relación entre los dos países a partir de la Revolución mexicana, la cual enfrentaría una serie de crisis a lo largo de la década. Para 1926, el deterioro en las relaciones bilaterales fue tan evidente que las energías de las mujeres panamericanistas ahora fueron dirigidas hacia una meta común: evitar una nueva guerra entre Estados Unidos y México.

A mediados de la década de 1920, la amenaza de intervención militar estadunidense tenía diversas causas, pero la más importante, que sería reflejada de manera explícita en Flame of Mexico, fue la cuestión del petróleo. Durante la presidencia de Plutarco Elías Calles, surgieron de nuevo los conflictos en torno al Artículo 27 de la Constitución, ya que

26 Anna Macías, Contra viento y marea. El movimiento feminista en México hasta 1940, pp. 122-125; también véanse las “Conclusiones del Primer Congreso Feminista de la Liga Panamericana de Mujeres", en Resoluciones tomadas en el Primer Congreso Feminista, 20 a 30 de mayo de 1923 (México, Talleres Linotipográficos "El Modelo", 1923), texto reproducido en Tunón (comp.), Voces a las mujeres, pp. 248-259.

27 Además de la correspondencia resguardada en la Library of Congress (en adelante LOC), Smith College (Margaret Sanger Papers) y Dartmouth College (Juliet Rublee Collection), véase Jane Elkind Bowers, “Oceans of Love': An Introduction to \& Excerpts from Juliet Barrett Rublee’s Letters to Margaret Sanger”, tesis de maestría, University of Texas at Austin, 1994.

28 Megan Threlkeld, “The Pan American...”, op. cit., p. 821; Ana LauJaiven, "Entre ambas fronteras: la búsqueda de la igualdad de derechos para la mujeres", Política y Cultura, p. 242. Véase también Julia Nava de Ruisánchez, "Carta Alocución Pronunciado en el Club Latino Americano de Baltimore, la noche del 21 de abril de 1922" (fragmento), en Tunón (comp.), Voces a las mujeres, p. 246. En este texto, Nava de Ruisánchez aclara que, aunque a las mujeres mexicanas les preocupaba el voto, su prioridad en ese entonces era la educación, la cual consideraban necesaria para el pleno ejercicio de sus derechos como ciudadanas. 
no se había resuelto el problema de la soberanía nacional en torno al subsuelo. Mientras el presidente Calles intentó promulgar una legislación limitando los poderes de las compañías petroleras extranjeras operando en México -en particular, las concesiones ilimitadas que habían sido otorgadas durante el Porfiriato-, las empresas se negaron a cumplir con las nuevas estipulaciones (contratos temporales y condicionados), argumentando que el gobierno mexicano estaba infringiendo sus derechos y violando la libertad de comercio establecida en acuerdos anteriores. En el congreso estadounidense, la influencia de las compañías petroleras en la política se tradujo en llamados, cada vez más estridentes, a la guerra.

Como respuesta, las mujeres panamericanistas organizaron, en contra de la amenaza de intervención, la "Campaña de paz con México" [Peacewith México Campaign] para movilizar la opinión pública a favor de la paz. Fomentaron la elaboración de cartas, editoriales periodísticas, telegramas y llamadas telefónicas al presidente Calvin Coolidge, además de sermones predicados por clérigos en Año Nuevo (táctica importante para contrarrestar el sentimiento de indignación en torno a la Guerra Cristera por parte de un sector de católicos estadunidenses), mítines masivos y otras estrategias para demostrar la amplitud del sentimiento público a favor de la paz; para enero de 1927, más de 10 millones de ciudadanos estadunidenses firmaron mensajes en contra del envío de tropas a México ${ }^{29}$. Aunque, como bien dice Threlkeld, la acción de las agrupaciones pacifistas no fue el único factor en detener la intervención militar en el país vecino -ya que la diplomacia también jugaría un papel central en ello-, seguramente la muestra del rechazo masivo a la guerra fue tomada en cuenta por el Congreso y por el presidente en su toma de decisiones sobre el asunto. De esta manera, las mujeres pacifistas ensayaron enérgicas estrategias propagandísticas y demostraron su fuerza política como bloque ciudadano significativo: fuerza que también intentará movilizar Juliet Rublee en su propia campaña, ahora cinematográfica, a favor de la paz entre las dos naciones.

\section{Flame of Mexico: el discurso amoroso como mensaje políti- co}

$\mathrm{Al}$ escribir el guion para Flame of Mexico, Rublee se nutría de varias tradiciones representacionales: en primer lugar, de la del cine de viajeros, una especie de medio didáctico que gozaba de gran popularidad entre 29 Megan Threlkeld, Pan American..., op. cit., pp. 118, 132-133, 135. 
el público ilustrado a principios del siglo XX, ya que ofrecía a los espectadores la posibilidad de "visitar" lugares lejanos, a menudo "exóticos", por medio de la cámara y de la narración en vivo del viajero-presentador. Sin embargo, aunque Rublee al principio concibió su película dentro de esta tradición, e incluso filmó varias escenas de gran belleza paisajista en colaboración con el geógrafo y fotógrafo capitán John Noel (famoso por fotografiar el ascenso de George Mallory a la montaña Everest), posteriormente decidió que un filme de ficción, con drama, romance y acción, sería la manera más efectiva de llegar a una mayor cantidad de espectadores $^{30}$.

Por lo tanto, filmó una narrativa que no sólo tomó elementos del cine dominante hollywoodense de la época -ideada y establecida poco antes por innovadores directores como D. W. Griffith y continuada en las películas de los 1920, entre las cuales se encuentran varias obras dirigidas por David Kirkland, director de Flame of Mexico-, sino que también prefiguraba el estilo de muchos melodramas del cine mexicano -entonces naciente como industria, pero que pronto entraría a su llamada Época de Oro- con su contenido ligado a la historia reciente de la Revolución, su narrativa de amor que trasciende clases sociales, su mirada crítica hacia el gringo explotador y su uso de la música como elemento predominante en la creación de significados.

Flame of Mexico es, sin duda, una historia de amor. El amor triunfa en la película, pero a diferencia de su representación en las películas mexicanas y estadounidenses contemporáneas, este amor no está destinado a desplazar otros conflictos con la promoción de la pareja como fin en sí (con las implicaciones correspondientes en torno al matrimonio y a la procreación), sino, primordialmente, a atraer al público cinéfilo, especialmente al norteamericano. A través de la música e imágenes de un México un tanto estereotipado, Rublee buscaba cambiar la opinión de los estadounidenses, que veían al país vecino a través de estereotipos negativos fomentados por el cine hollywoodense desde la era del cine mudo. La meta de Rublee era, sobre todo, convencer a la clase media estadounidense de que México era un país de gran belleza geográfica, con un pasado legendario y un presente en el que la cultura milenaria estaba todavía viva y en ebullición. Una parte esencial de su estrategia fue el uso de ciertas músicas regionales mexicanas, difundidas en aquel momento a través de la radio y de una naciente industria discográfica transnacio-

30 Isabel Arredondo, "From Travelogues to Political Intervention in Juliet Barrett Rublee's Flame of Mexico", Mexican Studies/EstudiosMexicanos, pp. 79-93. 
nal, como gancho para atraer al público; sin embargo, esta música, en la que domina el tema del amor romántico, no está destinada a asentar un sistema patriarcal, sino a asentar relaciones "amorosas" entre México y Estados Unidos con el fin de evitar sentimientos hostiles y bélicos, además de sus probables consecuencias políticas.

En Flame of Mexico se trazan dos narrativas que avanzan paralelamente: la del amor y la de la política. La función más obvia de la banda sonora es servir de diálogo amoroso, pero además busca seducir al público estadounidense (mostrándole un repertorio de música regional mexicana relativamente desconocida en Estados Unidos) y al público mexicano (a través de escenas emotivas en las que la música contribuye a crear diversas sensaciones en las vísceras del espectador). Cabe preguntarse si las múltiples escenas de persecución, que constituyen una parte significativa de la película y en las que la música es un elemento esencial, estaban destinadas a mostrar la virtuosidad de la imagen sonora en movimiento al público mexicano, al mismo tiempo que las escenas más folclóricas (tehuanas, fiestas patronales) construyeron una imagen pintoresca de México para las audiencias en Estados Unidos. Desde el punto de vista musical, en todo caso, se establece un diálogo musical intercultural e híbrido, con elementos puramente mexicanos y otros típicamente estadounidenses.

Cabe mencionar que la película comienza y acaba con un marco visual y narrativo refiriéndose a una leyenda de amor entre los volcanes Popocatepetl e Iztaccihuatl, con el acompañamiento de la canción Amorcito consentido en voz de Tito Guízar. Esta escena inicial, proponemos, busca seducir al público norteamericano con la belleza geográfica de los volcanes y el exotismo del mito romántico. La historia de los dos volcanes anticipa el tema del amor de los protagonistas y el papel de la música como elemento de diálogo amoroso. Además, imágenes del volcán Popocatepetl acompañarían al protagonista masculino a lo largo de la cinta, subrayando sus cualidades de héroe masculino y su estrecha conexión con la tierra y con la nación.

La historia de amor, política y seducción (entre los personajes y al espectador) que narra la película es a grandes rasgos la siguiente: Rafael (Donald Reed), de extracto pobre y campesino, presencia la muerte de su padre y recibe de él, en su lecho de muerte, sus consejos de tener fe en su pueblo, trabajar y estudiar para poder guiarlo, con amor y sabiduría, hacia la luz. Cumpliendo con el deseo de su progenitor, Rafael aprende a 
leer, estudiando en la noche después de sus largas jornadas de labor en la plantación donde gana 20 centavos al día. Con los primeros titubeos del movimiento maderista, Rafael se postula como candidato a diputado local por el "Partido Mexicano Progresista", y antes y durante su discurso se escucha el sonido diegético de una banda de viento ${ }^{31}$. Su contrincante, del "Partido Conservador Nacional", es don Gonzalo de Aragón (Francisco Martínez), hacendado que representa la oligarquía tradicional y que a la vez tiene negocios turbios con el empresario estadunidense Thornton (Frank Hagney) y con el hábil secuaz de éste, Fry (Jimmy Hodges). Don Gonzalo es también el padre de Rosita (Alicia Ortiz), la hermosa joven que es el objeto del deseo romántico de Rafael.

A pesar de su posición social como hija del hacendado, es evidente que Rosita también comparte el sentimiento amoroso, pues cuando Rafael da su discurso político en la plaza pública, ella, a pesar de los comentarios negativos de su padre, lo mira con admiración, especialmente cuando él llega al clímax de su arenga: "Lo que el mundo necesita es más amor, amor, amor" ["What the world needs is more love, love, love"). Desde ahí es evidente que el discurso político no se puede separar del discurso amoroso, y que la relación entre Rafael y Rosita, además de romántica, se inspira en el vagamente compartido anhelo de un mundo mejor basado en exaltados principios de justicia social y amor a la humanidad.

El romance entre Rafael y Rosita, igual que los sueños compartidos de justicia social, se encuentran interrumpidos por las maniobras de Thornton, quien comenta a Fry en la siguiente escena que ha prestado fuertes cantidades de dinero a don Gonzalo, y que para cobrarlas va a apropiarse de una propiedad de éste, cuyo nombre "Waters of the Rainbow" [Aguas del Arcoíris] indica lo que tienen abajo: petróleo. Además, dice Thornton, está la hija Rosita, otro "premio" que piensa cobrar; con respecto a la oposición política representada por Rafael, Fry comenta que no se le debe tomar en serio, ya que "es el ejército quien decide las elecciones". Este diálogo se puede considerar parte medular de la narrativa política, ya que ilustra la actitud poco escrupulosa de los inversionistas

31 En la casa de Rublee en Cornish, New Hampshire, encontramos un álbum con recortes de periódico sobre una banda de guerra dirigida por el capitán J. E. Roach. Roach’s Banda Mexicana hacía giras por Estados Unidos entre 1908 y 1911; estaba compuesta por 50 músicos mexicanos que provenían de las bandas del ejército y de la policía. Incluía, además, cantantes hombres y mujeres, y bailarines. El repertorio consistía en piezas contemporáneas ("standards") y música tradicional mexicana. Ya que el álbum de recortes de periódico era probablemente de Rublee, es posible que ella ya estuviera interesada en la música mexicana en la década de 1910. Véase la entrada sobre la J. E. Roach Banda Mexicana MusicCollection en Archives UM, Universidad de Maryland: http://digital.lib.umd.edu/archivesum/actions.DisplayEADDoc.do?source=/MdU.ead.scpa.0031. $\mathrm{xml} \&$ style=ead (consultado el 17 de febrero de 2015). 
gringos en México, además de la poca seriedad con que se toma el movimiento opositor en sus inicios, dado el evidente poder del régimen porfirista.

Las secuencias que siguen son, en cambio, elementos más bien de espectáculo, aunque con cierta función narrativa también, ya que, primero, es Fry quien con su actitud prepotente provoca el incidente en el que los caballos del coche en el que está Rosita salen desbocados y Rafael tiene que salir tras ellos, cabalgando furiosamente, hasta que logra darles alcance y, segundos antes de que el coche se despeñe por un acantilado, lograr salvar a la heroína. En esta escena de acción, como en muchas otras de la película, la música, con una percusión cada vez más acelerada según la persecución se va haciendo más peligrosa, acompaña y da vida a la imagen en movimiento, siendo de esta manera un elemento esencial de lo que Gilles Deleuze llama "the movement-image" ${ }^{32}$. El exitoso rescate de Rosita, acompañado de violín, devuelve la tranquilidad al espectador, cuyas vísceras estaban encogidas por la persecución (como sugiere Jennifer Barker en su análisis del efecto de la música en el espectador, The Tactile Eye) y refuerza indudablemente el incipiente amor entre la pare$\mathrm{ja}^{33}$.

De regreso a la hacienda de don Gonzalo, Rosita toca una guitarra y canta Es tu amor, la canción que también hemos escuchado cuando Rafael la rescata ${ }^{34}$. Pero al mismo tiempo que Rosita se pierde en sueños románticos, don Gonzalo y Thornton negocian la ominosa transferencia de tierras. Don Gonzalo, retratado como honorable patrón con valores sólidos aunque conservadores (a diferencia de la actitud mercenaria de Thornton), protesta que no se puede quitar sus tierras a los indígenas; Thornton, mintiendo, le asegura que recibirán una buena recompensa.

Aquí, en una mezcla de cine documental o de viajeros y drama romántico, presenciamos la fiesta de San Isidro en la ranchería de Aguas del Arcoíris: la danza de moros y cristianos, los ritos llevados a cabo por 32 Gilles Deleuze, Cinema 1. The Movement-Image.

33 Jennifer M. Barker, The Tactile Eye: Touch and the Cinematic Experience.

34 El compositor de "Es tu amor" fue Jorge del Moral; es interesante notar que, según las investigaciones de Jacqueline Ávila, en los años treinta se consideraba a del Moral como representativo de un estilo "viril" y, por lo tanto, verdaderamente mexicano, en contraste al “afeminado" Agustín Lara; Ávila cita un artículo de El Universal Ilustrado de 1936 que opina: "Las canciones mexicanas verdaderamente populares tienen sexo masculino ... Los autores [como Lara] que han mixtificado el sabor nacional en la canción mexicana se han apartado del sabor hombruno, del paladeo macho de nuestros sones" (Ávila, "Los sonidos del cine: Cinematic Music in Mexican Film, 1930-1951", tesis de doctorado, University of California, Riverside, 2011, p. 210). Cuando Flame of México pone "Es tu amor" en boca de una mujer (Rosita), subraya con sutileza la idea del amor como una relación recíproca entre iguales, algo bastante contrario -de acuerdo con Anna María Fernández Poncela en Pero vas a estar muy triste y así te vas a quedar. Construcciones de género en la canción popular mexicana- a las representaciones predominantes del amor en la música popular de la época. 
la "hechicera" (según los títulos) o chaman del pueblo. En esta sección se escucha una marimba con xilófonos, que está acompasada al ritmo del baile que aparece en la imagen: otro momento en el que la película introduce música mexicana, en este caso como una especie de paréntesis folclórico en la narrativa que, no obstante, funciona para subrayar la conexión de los habitantes con la tierra a través del cumplimento del rito agrícola tradicional ${ }^{35}$.

A continuación se suceden, a paso rápido, una serie de secuencias paralelas que agilizan la narración, llevando al espectador varias veces entre la ranchería (en la que se trata un tema político) y la casa de Rosita (donde el tema es amoroso). En estas escenas también la música juega un papel esencial. Las celebraciones de San Isidro son interrumpidas por la llegada de Fry con los papeles, ordenando la desocupación, los cuales circulan de mano en mano hasta llegar por fin a alguien que sabe leer. Mientras los pobladores contemplan su triste suerte, hay un corte y vemos a Rosita rezando a la Virgen María; entra su empleada, que actuando de cómplice en los deseos de Rosita hacia Rafael, le trae un cartel de Rafael como candidato, un recuerdo que le da evidente placer. Afuera se oye un mariachi y Rosita piensa que la serenata es de Rafael; sin embargo, no es él sino Thornton quien, al sonido de Estrellita marinera de Guty Cárdenas, intenta ganar el afecto de la joven. El rito, enfadoso para Rosita pero que evidentemente complace a don Gonzalo, se interrumpe cuando las autoridades de la comunidad llegan a enfrentarse con Thornton. Le reclaman su territorio ya que, habiendo vivido en esas tierras desde tiempos inmemoriales, se consideran los legítimos dueños. Un poco después, en una acción paralela, Rafael le canta a Rosita su propia serenata junto a la ventana, la cual, a diferencia de la de Thorton, ella acepta gozosa.

Mientras don Gonzalo entra a la recámara de su hija, Rafael se marcha para ayudar a los comuneros en su enfrentamiento con Thornton. Asumiendo el liderazgo de la situación, Rafael les manda regresar a su fiesta, prometiendo que no se les va a expulsar de sus tierras; luego dice indignado a Thornton: "Dos tipos de extranjeros vienen acá: los que ayudan y los que nos hacen daño. iUsted es el tipo equivocado!" ${ }_{36}$ Este comentario, de cierto modo gratuito en la narrativa, ya que la película no propone ejemplos de extranjeros "buenos", refleja no obstante la perspectiva de la misma Juliet Rublee, abogada del panamericanismo y del feminismo en su concepto internacional. El internacionalismo, por de-

35 San Isidro Labrador lleva la lluvia a los sembradíos en su debido momento para asegurar una buena cosecha. 36 “Two kinds of strangers come here, those who help and those who hurt us. You冈re the wrong kind!" 
finición, propone la posibilidad de un relación positiva entre habitantes de diversas naciones y, por lo tanto, la posibilidad de una intervención positiva en México por parte de gente de buena voluntad (por ejemplo, las proponentes de la planificación familiar y los derechos de la mujer, o los embajadores cuya estrategia es mediar y no imponer ni aprovecharse de las debilidades políticas de un país en crisis). Rafael, por lo tanto, es nacionalista pero no xenofóbico y, además, por su amor a Rosita, está dispuesto a cruzar barreras de clase y efectuar alianzas para su propio bienestar y, primordialmente, el de su gente.

La intervención de Rafael resulta en un cruel castigo, en que el campesino será enterrado en la tierra hasta el cuello y aplastado por la caballería de los secuaces de Thornton. Cuando estos lo dejan por muerto, Rosita y su compañera lo desentierran a escondidas y lo llevan a una curandera. Dos semanas después, habiendo recuperado la salud, Rafael parte hacia Tierra Caliente, diciendo a Rosita: "Pronto México estará libre y feliz, y entonces quizás nosotros podamos ser felices también" ${ }^{37}$. Nuevamente, la felicidad personal de la pareja está ligada estrechamente a la libertad del pueblo mexicano. Sin embargo, lo que luego se ve en Tierra Caliente es desolador: los indígenas desplazados de Aguas del Arcoíris se ven forzados a trabajar en la hacienda, en condiciones de casi esclavitud. La banda sonora en la que se escucha la Canción mixteca refuerza la idea de que, en el exilio, uno vive no sólo en pésimas condiciones sino en un estado de tristeza permanente; desde la perspectiva histórica, también nos muestra que esta canción ya era el himno no oficial del mexicano desterrado.

Fry, en cambio, vive en esplendor colonial, rodeado de artefactos de tipo "Mexican curious" (esculturas, tapetes, etc.); cuando llega Thornton, dice con toda tranquilidad que Rafael ya está muerto, lo cual abre paso a la siguiente escena: la boda de Thornton y Rosita, acompañada por una extraña procesión de tehuanas que parece reflejar más la moda a la hora de hacer la película que las verdaderas prácticas nupciales de las haciendas en el sur de México, ya que para la década de 1920, las tehuanas, mujeres zapotecas del Istmo de Tehuantepec, conocidas por su indumentaria elaborada, su joyería, su belleza y su fortaleza física, ya se habían convertido en uno de los símbolos nacionales más atractivos y exóticos, tanto para el público mexicano como para el extranjero. Como sea, la joven se ve resignada; nada le importa, ya que su amado ha muerto; pero apenas efectuada la ceremonia, aparece Rafael desde la selva 37 "Soon México will be free and happy, and then perhaps we can be happy as well." 
para liderar, nuevamente, la rebelión, y claro, el amor de Rosita.

En medio de este escenario, llega a la hacienda un telegrama anunciando los inicios de la Revolución en el norte y avisando que todos los extranjeros deben salir de inmediato del país. Mientras Thornton y Fry deciden huir a la Ciudad de México, don Gonzalo y Rafael hacen las paces, aquél explicando a éste que no sabía qué clase de hombre era Thornton. Rafael le ofrece al hacendado la protección de sus hombres para ir a la capital. Estalla la rebelión de verdad y hay un salto en el tiempo al maderismo, criticado por Rafael -como hicieron las fuerzas zapatistas en la vida real- por no cumplir sus promesas. Otro aspecto del maderismo es aludido en la escena: Rafael, ya en la ciudad capital, escucha las exhortaciones de un crítico del régimen y pregunta a un gendarme por qué no le impide hablar; el policía contesta que el nuevo gobierno ha dado órdenes de no impedir la libertad de expresión. Thornton, aparentemente consciente de los errores políticos del gobierno de Madero, mira los eventos con casi lasciva anticipación, ya que el régimen golpista de Victoriano Huerta -que sabemos de manera extratextual fue ayudado por el apoyo tácito del embajador estadounidense Henry Lane Wilson, y con el que en la película Thornton muestra complicidad- ya no impedirá su acceso a las tierras mexicanas y a su codiciado petróleo.

En el confuso segmento final de la película, para el que Rublee emplea noticieros de la Revolución que contienen bombardeos y batallas, vemos a Rafael en México, ahora vestido de traje al uso metropolitano, donde recibe el aviso de Rosita de un inminente golpe de Estado. La espía Thornton, pero ella lo desafía; al estar noqueada por una explosión, Rafael la salva una vez más, antes de reaparecer como político, siguiendo en la lucha progresista. "Rafael leads on the Progressives" dice uno de los títulos, utilizando un término propio a la política estadunidense para hacer legible su posición a una audiencia no mexicana; sin embargo, por sus seguidores campesinos y su lema de "iTierra y libertad!", se puede pensar que Rafael sigue luchando al lado de las fuerzas zapatistas, las más radicales entre las facciones revolucionarias en sus demandas por la tierra. Al mismo tiempo, un segundo lema, “iEstabilidad y progreso!", indica que la postura representada por Rafael está en perfecta armonía con las metas y valores del movimiento panamericano/internacionalista y que, lejos de ser una amenaza, representa el mejor futuro para México y, por lo tanto, para las relaciones políticas entre los dos países.

La película concluye, como buen melodrama, con el amor entre Ra- 
fael y Rosita en plena floración. Es de notar que además del traje que, como ya mencionamos, Rafael ya porta en lugar de su tradicional ropa de campesino, Rosita también ha cambiado: usa el corte de cabello moderno, "pelona", como se decía en la década de 1920, y la ropa moderna de una chica de la ciudad. Ya no es una mujer de hacienda, destinada a casarse con algún rico y vivir bajo su mando; es una mujer moderna y libre, preocupada con la educación ${ }^{38}$, que vive con la esperanza de cambiar su país al lado de su esposo en una relación de igualdad. En este sentido, a pesar del aparente cliché del amor que trasciende la diferencia de clases, no se parece en nada a los melodramas mexicanos de la Época de Oro, en que la narrativa gira en torno a la domesticación de una mujer "rebelde" a través del amor heteronormativo: por ejemplo, en Enamora$d a$, dirigido por Emilio Fernández en 1946, la joven mujer rica y altanera representada por María Félix se enamora del arrogante revolucionario (Pedro Armendáriz) y termina sacrificando sus bienes, estatus social e identidad personal para seguirle a "la bola" como soldadera. En cambio, el amor entre Rosita y Rafael, a pesar de las varias veces que éste tiene que ir al rescate de su amada para satisfacer las demandas de una película hollywoodense de acción, es una relación de iguales que se basa en la atracción romántica, pero también en la pasión compartida por ideales libertarios y la esperanza de participar, ambos, en la construcción de una nueva sociedad fundamentada en principios humanistas.

Esta sociedad, mientras cuenta con la bondad de figuras benévolas de la élite nacional como don Gonzalo, requiere imponer límites sobre la libertad de acción de extranjeros siniestros como Thornton y Fry, quienes han aprovechado las precarias condiciones de las masas mexicanas para extraer riquezas y apropiarse de lo que se les antoje (tierras, recursos, mujeres...), contando con la complicidad de las autoridades. Al mismo tiempo, la idea expresada por Rafael sobre los "dos tipos de extranjeros" rechaza el nacionalismo excluyente (xenofóbico) y en su lugar deja abierta la puerta a alianzas positivas, como las que se habían establecido en los congresos panamericanos de mujeres y que, a finales de la década de 1920, Rublee esperaba ver también en la esfera diplomática.

\section{Serenata transnacional: usos de la música en}

38 El hecho de que Rosita se convierte en maestra rural no es tan evidente en la película final como es en el guion, ya que en sus escritos se entiende que Rublee pensaba en el futuro de la protagonista como mujer moderna, liberada de las reglas del antiguo régimen patriarcal y colaboradora en el cambio social. 


\section{Flame of Mexico}

El mensaje que, como mostramos en el apartado anterior, se comunica en Flame of Mexico a través de una historia de amor y de acción, se refuerza de manera sobresaliente en las escenas que resaltan temas sonoros derivados de la música entonces popular en el país, debido a los medios emergentes, sobre todo la radio, pero también el cine, el cual había adquirido su "voz" precisamente en los años en que Rublee estaba en la producción de su película. En Estados Unidos, The Jazz Singer, de 1927, se considera la primera película sonora, aunque serían todavía unos años antes de que el sonido se incorporara plenamente y no de manera fragmentada a la producción cinematográfica. En el cine mexicano, el sonido aparece alrededor de 1931, con Santa, clásica obra de la literatura moderna y también del cine mudo, ahora filmado por el director Antonio Moreno, con música del compositor romántico Agustín Lara. A pesar de unos tanteos experimentales que se habían realizado en México desde 1929, Santa se considera "la primera cinta mexicana filmada con sonido óptico y la que inauguraría la etapa industrial en el cine mexicano" ${ }^{9}$. Dada esta estrecha temporalidad histórica, vemos que Rublee, como en muchos otros aspectos de su vida, estaba a la vanguardia cuando, cierto tiempo después de haber terminado Flame of México como película muda, decidió sincronizar el sonido para asegurar que las canciones mexicanas -ya incluidas en forma de partituras y de discos grabados- quedaran como parte medular del filme, el cual registró de nuevo para formalizar este avance tecnológico.

En un principio, parece que la influencia musical más importante sobre Rublee y Flame of Mexico fue el cantante y compositor yucateco Guty Cárdenas, a quien se atribuye las canciones en los créditos de la película, y con quien Rublee tenía correspondencia y, sin duda, algún contacto personal. Aunque es posible en teoría que Rublee conociera a Cárdenas y/o haya escuchado su música en su viaje a Mérida en 1923, en realidad el joven trovador aún estaba entonces en los inicios de su carrera; tocaba en conjuntos locales durante 1925 a 1927, año en que se mudó a la Ciudad de México y empezó a ganar premios por sus canciones, lo cual le abrió paso a la grabación. Entre abril de 1928 y septiembre de 1931 estuvo en Nueva York grabando para la Columbia Phonograph Company, hecho que hace pensar que quizás el contacto entre Rublee y Cárdenas tomó lugar no en México sino en Estados Unidos.

39 Maximiliano Maza, “Santa (1931)”, en Más de 100 años del cine mexicano. 
Sabemos, eso sí, que Rublee había escuchado a Cárdenas cantar en Hollywood, probablemente cuando ella estaba editando la película, ya que en una carta fechada el 29 de diciembre de 1931, el "Ruiseñor Yucateco" escribe que con gusto le daría permiso para utilizar cualquiera de las canciones de las que habían hablado entonces o bien cualquiera otra de su repertorio; agrega que le desea mucho éxito con la película, además de un feliz y próspero año nuevo. El asesinato de Cárdenas en la Ciudad de México el 5 de abril de 1932 ocurrió poco después del registro de Flame of México con banda sonora en enero del mismo año ${ }^{40}$; a pesar de este abrupto fin a la colaboración, la convergencia de dos trayectorias tan distintas es un accidente histórico interesante para nuestro examen de los lazos culturales transnacionales de la época, lazos tejidos y reforzados a través, en este caso, de la música y del cine ${ }^{41}$.

De hecho, gracias a la antemencionada carta, sabemos que fue Cárdenas quien tradujo y arregló la canción principal ("themesong" en los créditos) de la película, Love Never Dies de la autoría de Charles Dixon. Cantada en español, es esta la pieza que lleva Rafael a Rosita como serenata, cuando el joven político y activista campesino está en campaña. En su recámara, Rosita está admirando la imagen de Rafael plasmada en el cartel del Partido Progresista, cuando llega, primero, la serenata enviada por Thornton (Estrellita marinera, también de Cárdenas); al terminar este gesto indeseado por parte de su recipiente, llega Rafael montado a caballo, cantando (cabe subrayar que mientras Thornton depende de los mariachis, Rafael lleva la canción con su propia voz):

Dulce bien, pronto a mis brazos...

Escúchame, ten piedad de mí

Quiero cantar, mi canción de amor...

Como ya hemos señalado, en boca de Rafael el asunto de "amor" tiene dos significados: su sentimiento romántico para Rosita, que es el significado que predomina aquí, pero al mismo tiempo, "amor" como el noble sentimiento que inspira su lucha política y trasciende la esfera personal para convertirse en el sueño y apoteosis de la nación. Es por eso que la 40 En el registro de derechos de autor de la LOC se encuentra la siguiente entrada: "Soul of México, sound picture, musical acc. without words, by Nilo Menéndez, of Cuba and Arnulfo Miramontes, of México, both domiciled at New York". Esta versión musicalizada de la película queda registrada el 30 de enero de 1932 a nombre de Rublee (Catalog of Copyright Entries: Musical Compositions, Part 3, LOC Copyright Office, 1932); el título Soul of Mexico quizás representa una exigencia legal ya que la misma película cuenta con un registro anterior. 41 Cárdenas incluso había participado en una película de la productora First National, La dama atrevida, dirigida por William C. McGann y Guillermo Prieto Yeme en 1930 y estrenada en 1931. 
misma canción se repite al final de la película, cuando el binomio Popocatepetl-Iztaccihuatl constituye el fondo para el "final feliz", aparentemente convencional, de reconciliación y unión romántica entre la pareja protagonista. Este aparente convencionalismo, dado el doble juego encarnado en la canción El amor nunca muere, resulta ser superficial, ya que el mensaje más profundo tiene que ver no sólo con el romance heteronormativo del melodrama sino, y de manera más importante, con el sueño panamericano de la unidad y la armonía entre las naciones.

Entre los otros músicos que colaboraron con Rublee, destaca la presencia del cantante Tito Guízar, estrella de la radio bilingüe en Estados Unidos en aquel entonces, y por supuesto, futuro protagonista de Allá en el rancho grande (Fernando de Fuentes, 1936) y muchas otras obras del cine mexicano y hollywoodense. También llama la atención la inclusión de la Canción mixteca del oaxaqueño José López Alavés, con quien Rublee trató directamente para solicitar los derechos ${ }^{42}$. Esa famosa canción, alusiva a la época revolucionaria y sus vaivenes, fue escrita en 1915 y presentada en la Ciudad de México en 1918 como ganadora de un concurso de canción. Como ya hemos mencionado, en Flame of México -posiblemente la primera película de muchas $^{43}$ en las cuales aparece este clásico lamento del campesino desterrado la Canción mixteca acompaña las escenas de los indígenas desplazados de su ranchería Aguas del Arcoíris y trabajando forzosamente en la hacienda de Tierra Caliente. De esta manera, los poderosos versos alusivos a la soledad y a la tristeza sirven para matizar la narrativa y subrayar su historicidad.

Con este breve recorrido de las canciones y su uso como elemento narrativo y expresivo en la película, nos damos cuenta, en primer lugar, del enorme flujo transnacional de expresiones musicales y, en particular, de la íntima relación entre la industria discográfica basada en Estados Unidos (principalmente en ese entonces en Nueva York) y el florecimiento de las músicas regionales mexicanas y su reinvención como música "nacional" durante la época posrevolucionaria ${ }^{44}$. Por otra parte, es notable el tino de Juliet Rublee, en este aspecto, al escoger para su ban42 En la LOC hay cartas que nos permiten saber que Rublee pidió los derechos de la canción a López Alavés y que éste se los concedió el 8 de septiembre de 1930.

43 Un pequeño recorrido por la trayectoria de esta canción en el cine se encuentra en el blog Urruneando el 6 de abril de 2014, con el título “Canción mixteca... de cine..., sin firma de autor: http://urruneando.blogspot. mx/2014/04/cancion-mixteca.html (consultado el 9 de febrero de 2015). La misma canción aparece en Allá en el Rancho Grande, donde sus connotaciones son, por supuesto, distintas.

44 Tema explorado por Ricardo Pérez Montfort en trabajos como Estampas de nacionalismo popular mexicano: ensayos sobre cultura popular y nacionalismo; Avatares del nacionalismo cultural: cinco ensayos; Expresiones populares y estereotipos culturales en México, siglos XIX y XX: diez ensayos; $y$ Cotidianidades, imaginarios $y$ contextos: ensayos de historia y cultura en México, 1850-1950. 
da sonora artistas e incluso canciones que se incluirían, de la década de 1930 en adelante, en el reportorio básico del cine mexicano en su Época de Oro. Es decir, lejos de ser la dama rica y bienintencionada pero poco familiarizada con la cultura mexicana retratada en la crítica, está claro que Juliet poseía una familiaridad con la música de su época que iba más allá de lo meramente turístico: o sea, a pesar de ciertos elementos quizás demasiado vistosos y folclóricos para ser creíbles como parte de la narrativa (la procesión de las tehuanas en la boda de Rosita y Thornton, por ejemplo), está claro que ella tenía plena consciencia de los usos de la cultura, y también de sus abusos, como hemos señalado en la escena de Fry rodeado de objetos tipo "Mexican curious" mientras explota la labor de los peones en la hacienda. De los "dos tipos de extranjeros" aludidos por Rafael en la película, Rublee, a diferencia de sus personajes Thornton y Fry, se posiciona como alguien dispuesta a escuchar, observar, entablar amistades y, en última instancia, apoyar y compartir visiones de justicia social y humanitarias cuyas raíces e implicaciones son tanto nacionales como universales.

\section{Reflexiones finales}

La década de 1920 fue un tiempo intenso para las relaciones políticas y culturales entre México y Estados Unidos, tanto las oficiales como las no oficiales, ejemplificadas en la obra de los muchos viajeros estadounidenses que dejaron testimonios de sus experiencias e impresiones del vecino país ${ }^{45}$. Más o menos conocido es el grupo de intelectuales que, durante varios años, se dedicaba a difundir más allá de las fronteras nacionales las tradiciones artísticas y también las nuevas ideas revolucionarias emanadas de México: Frances Toor, directora de la revista Mexican Folkways; Anita Brenner, nacida en México pero educada en Estados Unidos, autora del libro fundamental Ídolos tras las altares (1929) con colaboraciones de los fotógrafos Edward Weston y Tina Modotti, y posteriormente de The Wind that Swept México (1943) y Your Mexican Holiday (1947); Ernest Gruening, prominente miembro -como Rublee- del movimiento en pro del control de la natalidad y a la vez estudioso de los procesos históricos mexicanos; el escritor comunista Joseph Freeman, quizás el crítico más severo de las posturas trotskistas de Diego Rivera y de las colaboraciones de éste con el gobierno estadounidense a través de

45 Véase Helen Delpar, The Enormous Vogue of Things Mexican: Cultural Relations Between the United States and Mexico, 1920-1935. 
Dwight Morrow; y el también escritor y periodista Carleton Beals, autor de Mexican Maze (1931), entre otros.

Beals, a pesar de ser más moderado que Freeman en términos de sus afinidades políticas, también fue una figura controvertida debido a su fuerte crítica de la postura intervencionista de Estados Unidos en México y en otros países latinoamericanos ${ }^{46}$. En el Segundo Congreso sobre la Causa y la Cura de la Guerra [Second Conference on the Cause and Cure of War], realizado en diciembre de 1926, Beals impartió un apasionado discurso que ofendió a la organizadora principal del Congreso, la internacionalista moderada Carrie Chapman Catt (conocida también por su activismo en la lucha por el sufragio femenino). Beals atacó las políticas norteamericanas en México por tener en el fondo la avaricia de por la tierra y por recursos como el petróleo; en particular, criticó al presidente Woodrow Wilson y al embajador Henry Lane Wilson (cómplice, como hemos mencionado, del golpe huertista de 1913) por haber "derramado un río de sangre sobre la historia de México" ${ }^{47}$. Catt, por temor a represalias de parte del gobierno de Wilson contra los patrocinadores del Congreso, terminó borrando los comentarios de Beals del récord oficial del evento ${ }^{48}$.

A pesar de este acto de censura que muestra la gravedad del conflicto sobre México a mediados de la década, la investigación de Threlkeld constata que la perspectiva de Beals, en contra de la intervención político-militar y a favor de la paz y la cooperación, fue compartida por gran parte de la población estadounidense de la época, la cual desde 1922 incluía a las mujeres como ciudadanas con derecho de participar y opinar, tanto en asuntos domésticos como en los internacionales. Tanto los congresos feministas como las organizaciones a favor de la paz, lideradas en gran parte por mujeres, muestran la importancia del activismo femenino como factor en la determinación de la opinión pública en torno a México. En este sentido, la película de Rublee fue parte de un fenómeno amplio que surgió después de la Revolución con el fin de evitar confrontaciones (como la ocupación del puerto de Veracruz en 1914 o la "Expedición Punitiva" contra Pancho Villa en 1916) y promover la paz.

La favorable retroalimentación que recibió Flame of México de

46 La intervención estadounidense en Nicaragua fue otro tema que afectó las relaciones entre México y Estados Unidos debido a sus distintas perspectivas en torno a la nación centroamericana, o sea, la negativa por parte de México de seguir ciegamente las políticas dictadas desde Washington. Aunque esta historia rebasa los límites del presente trabajo, forma parte del contexto político del panamericanismo y de la producción de Flame of México.

47 Megan Threlkeld, Pan American..., op. cit., pp. 124-125.

48 Ibid., p. 125. 
parte de muchos miembros de los sectores intelectuales y diplomáticos ${ }^{49}$ señala que su proyecto logró comunicar su mensaje; por lo tanto, es importante retomarlo no sólo como parte de la historia cinematográfica, sino también como un ejemplo del activismo feminista de su productora, que toma posición frente a las tenues relaciones binacionales a través de un arte que agrada y seduce (con su música y su acción) y que cuestiona (a través de su narrativa) la posición de Estados Unidos frente a la Revolución mexicana, exigiendo el respeto a la soberanía del pueblo mexicano sobre su territorio.

Flame of México, primero y último largometraje de Juliet Barrett Rublee, resulta ser una película híbrida en muchos de los sentidos de la palabra: al ser una de las primeras -si no la primera- cintas de ficción filmadas por productores estadunidenses en México, es una película que literalmente atraviesa fronteras, asimilándose a la narrativa nacional mexicana con el fin no de participar en la industria nacional, sino de llevar una imagen verosímil de México al público estadunidense. Además, resalta en ella la vigorosa participación de músicos quienes estaban en el proceso de desarrollar sus carreras de por sí híbridas, reinventando géneros musicales regionales mexicanos para su adaptación a los nuevos medios como la radio y el cine, además grabando y presentándose en vivo en un circuito transnacional que incluía, entre sus polos más relevantes, a la Ciudad de México, Nueva York, Los Ángeles y La Habana. Avalada por estos diversos recursos humanos y comunicacionales, la película de Rublee barre las fronteras entre documental y ficción, entre melodrama, película de viajeros y -de manera más sutil- argumento político.

Además, después de hacer su película con títulos escritos al estilo del cine mudo, al presenciar el fin de éste, Rublee hace el intento de entrar a la nueva época de cine sonoro, agregando una banda sonora que termina siendo un elemento esencial de la narrativa. Hay que aclarar, sin embargo, que antes de la advenimiento del cine sonoro en el sentido de la fusión entre imagen y sonido en el material óptico de la película proyectada, las cintas podían llevar acompañamiento musical a través de grabaciones o, más comúnmente, música en vivo interpretada por pianistas, pequeños conjuntos o grandes orquestas de acuerdo con el tamaño y nivel económico de la sala de cine. Por lo tanto, consideramos que las escenas musicales de Flame of Mexico siempre fueron pensadas como "sonoras"; de hecho, aun cuando se había registrado la banda so-

49 Consta esta retroalimentación en las cartas y otros documentos localizados en los archivos de Rublee, en contraste con la escasa mención de Flame of México en las historias y recopilaciones del cine de los 1920 y 1930. 
nora a principios de 1932, los documentos indican que Rublee contrató a los cubanos Ignacio y Mercedes Ruffino, futuros fundadores del famoso Cuarteto Ruffino, para tocar como entretenimiento adicional en (antes y/o después) las presentaciones de la película en el Rialto Theatre en Washington, DC, empezando el 3 de junio del mismo año. Ya que los Ruffino estaba en los inicios de su exitosa carrera -cantarían en películas hollywoodenses y mexicanas durante los años cuarenta-, una vez más es de apreciar el instinto de Rublee para promover talentos de calidad, con el potencial de atraer a audiencias amplias, aun cuando, irónicamente, su propia producción fílmica no recibiera el beneficio de este talento.

Entre cine silente y sonoro, mexicano y hollywoodense, narrativo y documental, romántico y político, didáctico y de entretenimiento, Flame of Mexico resulta ser un artefacto sui generis en la historia cultural de ambos países. Su falta de éxito comercial -en parte por su propia condición de hibridez, y en otra gran parte por la crisis económica con la cual coincidió su conclusión y estreno, y la cual, junto con las nuevas tensiones en Europa, eclipsó en el imaginario social norteamericano la importancia de las relaciones interamericanas y la paz con México- no justifica su desconocimiento (cuando no menosprecio) por parte de la crítica; tampoco su notoria ausencia de la historiografía del cine de la época, que ha exaltado otros proyectos transnacionales que, aunque plagados de obstáculos en su momento, ahora son legendarios, como Que viva México de Eisenstein o Redes de Fred Zimmerman, sin reconocer en el proyecto de Rublee un importante y cercano antecedente. Como mujer, feminista, aventurera y libre pensadora, Juliet Rublee quizás no logró que se tomara en serio su trabajo como cineasta; sin embargo, su serenata transnacional por la paz permanece como ejemplo del impulso panamericanista y del intento de usar el cine como vehículo de comunicación en pro de la diplomacia y de la ideología internacionalista de los complicados años veinte.

\section{Bibliografía}

ARREDONDO, Isabel. "From Travelogues to Political Intervention in Juliet Barrett Rublee's Flame of Mexico”, Mexican Studies/Estudios Mexicanos. Vol. 26, núm. 1, invierno, 2010, pp. 79-93.

ÁVILA, Jacqueline. Los sonidos del cine: Cinematic Music in Mexican 
Film, 1930-1951. Tesis de doctorado, University of California, Riverside, 2011.

BARKER, Jennifer M. The Tactile Eye: Touch and the Cinematic Experience, Berkeley/Los Angeles, University of California Press, 2009.

BOWERS, Jane Elkind. 'Oceans of Love': An Introduction to \& Excerpts from Juliet Barrett Rublee's Letters to Margaret Sanger. Tesis de maestría, University of Texas at Austin, 1994.

BUCK, Sarah A. "El control de la natalidad y el día de la madre: política feminista y reaccionaria en México, 1922-1923”, Signos Históricos. Núm. 5, enero-junio, 2001.

"Canción mixteca... de cine...", Urruneando, 6 de abril de 2014: http:// urruneando.blogspot.mx/2014/o4/cancion-mixteca.html (consultado el 9 de febrero de 2015).

CHESLER, Ellen. Woman of Valor: Margaret Sanger and the Birth Control Movement in America. Anchor Books/Doubleday, Nueva York, 1992.

DELPAR, Helen. The Enormous Vogue of Things Mexican: Cultural Relations Between the United States and Mexico, 1920-1935. University of Alabama Press, Tuscaloosa, 1992.

DELEUZE, Gilles. Cinema 1. The Movement-Image. Trad. De Hugh Tomlinson y Barbara Habberjam, University of Minnesota Press, Minneapolis, 1986.

FERNÁNDEZ PONCELA, Anna María. Pero vas a estar muy triste y así te vas a quedar. Construcciones de género en la canción popular mexicana. Conaculta e INAH, México, 2002.

GARCÍA RIERA, Emilio. Historia documental del cine mexicano. Tomo 1, Universidad de Guadalajara/Gobierno de Jalisco/Conaculta/Imcine, Guadalajara, 1992.

GRUENING, Ernest. Mexico and its heritage. The Century, Nueva York, 1929.

LAUJAIVEN, Ana. "Entre ambas fronteras: la búsqueda de la igualdad de derechos para la mujeres", Política y Cultura. Núm. 31, primavera, 2009, pp. 235-255.

LEMAÎTRE, Monique J. Elvia Carrillo Puerto. La Monja Roja del Mayab. Castillo, Monterrey, 1998.

Library of Congress. Copyright Office. Catalog of Copyright Entries: Musical Compositions, Part 3, LOC Copyright Office, 1932. https:// archive.org/details/catalogofcopyrig253libr 
MACÍAS, Anna. Contra viento y marea. El movimiento feminista en México hasta 1940. México, Programa Universitario de Estudios de Género, Coordinación de Humanidades, UNAM, 2002.

MARASHIO, Paul. "A Feminist Voice in New Hampshire”. Texto inédito, Juliet Rublee Papers, Rauner Special Collections, Dartmouth College, Hanover, NH, 1982.

MAZA, Maximiliano. “Santa (1931)”, en Más de 100 años del cine mexicano, 1996: http://cinemexicano.mty.itesm.mx/peliculas/santa1. html (consultado el 9 de febrero de 2015).

PÉREZ MONTFORT, Ricardo. Cotidianidades, imaginarios y contextos: ensayos de historia y cultura en México, 1850-1950. CIESAS, México, 2008.

. Expresiones populares y estereotipos culturales en México, siglos XIX y XX: diez ensayos. CIESAS, México, 2007.

. Avatares del nacionalismo cultural: cinco ensayos. Centro de Investigación y Docencia en Humanidades del Estado de Morelos, México, 2000.

Estampas de nacionalismo popular mexicano: ensayos sobre cultura popular y nacionalismo. CIESAS, México, 1994.

RASHKIN, Elissa e Isabel Arredondo. "Juliet Barrett Rublee y la Revolución mexicana”, en Susie Porter (coord.), Género en la encrucijada de la historia social e cultural. El Colegio de Michoacán y CIESAS-Occidente, Zamora/México, en prensa.

SANGER, Margaret. The Autobiography of Margaret Sanger. Mineola, NY, Dover, 2004.

THRELKELD, Megan. Pan American Women: U. S. Internationalists and Revolutionary Mexico. University of Pennsylvania Press, Philadelphia, 2014.

- "The Pan American Conference of Women, 1922: Successful Suffragists Turn to International Relations”, Diplomatic History. Vol. 31, núm. 5, 2007, pp. 801-828.

TUÑóN, Julia (comp.). Voces a las mujeres. Antología del pensamiento feminista mexicano, 1873-1953. Universidad Autónoma de la Ciudad de México, México, 2011. 